\title{
Postgraduate teaching in clinical genetics in the United Kingdom
}

\author{
A W Johnston
}

The explosion of information in clinical genetics over the past decade stimulated an enquiry into the teaching of clinical genetics in the United Kingdom. A survey was first undertaken of the timetabled teaching in the British medical schools. The findings and subsequent recommendations were published as a report from the Royal College of Physicians in $1990 .{ }^{1}$ Since there are many more doctors than medical students, data on postgraduate teaching were also essential. The postgraduate deans of each medical school were approached first and later the help of a local clinical geneticist was sought.

\section{Survey of postgraduate deans (Directors of Education)}

The initial survey took the form of a short questionnaire asking about the teaching of clinical genetics in postgraduate programmes. It was circulated to all postgraduate deans in April 1990. The principal objective was to try to ascertain the number of hours devoted in 1989 to lectures, seminars, etc in the four different postgraduate programmes for general practice, general (internal) medicine, paediatrics, and obstetrics. Only five of the 24 deans could provide much information, which they had usually obtained from several of their postgraduate tutors (or equivalent). Yet, even here, no information was recorded for approximately a quarter of the possible entries on the questionnaire. Out of the total of 43 replies, only four deans reported a total of seven groups who received six or more hours of lectures. The seven groups came from all four disciplines. Several deans commented specifically on the difficulty of obtaining this type of information, so that they had no overall view. Nine stated that no information was available, including two with internationally known genetic centres! Information from deans was clearly too scanty to provide any reliable data on the extent of postgraduate teaching, and we were unable to discern any systematic approach to postgraduate teaching in general.

It is perhaps worth noting some of the comments about genetics from the deans. These varied from awareness to ignorance; for example, "a high priority" to "until this questionnaire came, the subject of genetics as a separate discipline had not actually raised itself in my mind ... (I will) give it some thought".
A more appropriate comment was that the "JRCP paper (on teaching genetics to medical students) and the questionnaire are a timely reminder of a relevant subject with important new advances". Perhaps the mere sending of the questionnaire was in itself an educative exercise!

\section{Survey of clinical geneticists}

Because so little information was obtained from deans, a second survey was carried out using (usually) one consultant clinical geneticist in each region, and including Wales, Ulster, and the four Scottish teaching hospitals. Every region, except two without a consultant clinical geneticist, replied and, with only one or two exceptions, provided considerable detail. The questions were extended to include lectures to nurses and other groups, such as lay organisations, as it was known that some centres were involved in this way. Information on who delivered the lectures was also sought. For the purposes of analysis, Trent is counted as three centres based on the medical schools. Replies were, therefore, available for analysis from 20 centres. The abstracted data were first sent back to respondents to be verified and they were asked to be particularly careful to ensure that all lectures, including those to nursing, paramedical, and lay groups, were included in their totals. Perhaps the most striking feature is the marked variation in the number of lectures given to all groups and reported by the different centres. The lowest number given was six hours where there was no consultant clinical geneticist, and 14 hours where there was a clinical geneticist, while the maximum was 151 hours.

\section{Status of lecturer and lectured}

Most lectures were given by the clinical geneticists, though with some support from nursing and scientific staff. An average of 36 hours was given to medical staff in the four disciplines surveyed. However, this covers a very wide range, from one centre giving less than 10 hours to four where between 80 and 99 hours per annum were delivered (figure). Within the four disciplines, there was a fourfold difference between some centres. More striking still was the tenfold variation in the number of Infirmary, Aberdeen AB9 2YS.

A W Johnston 


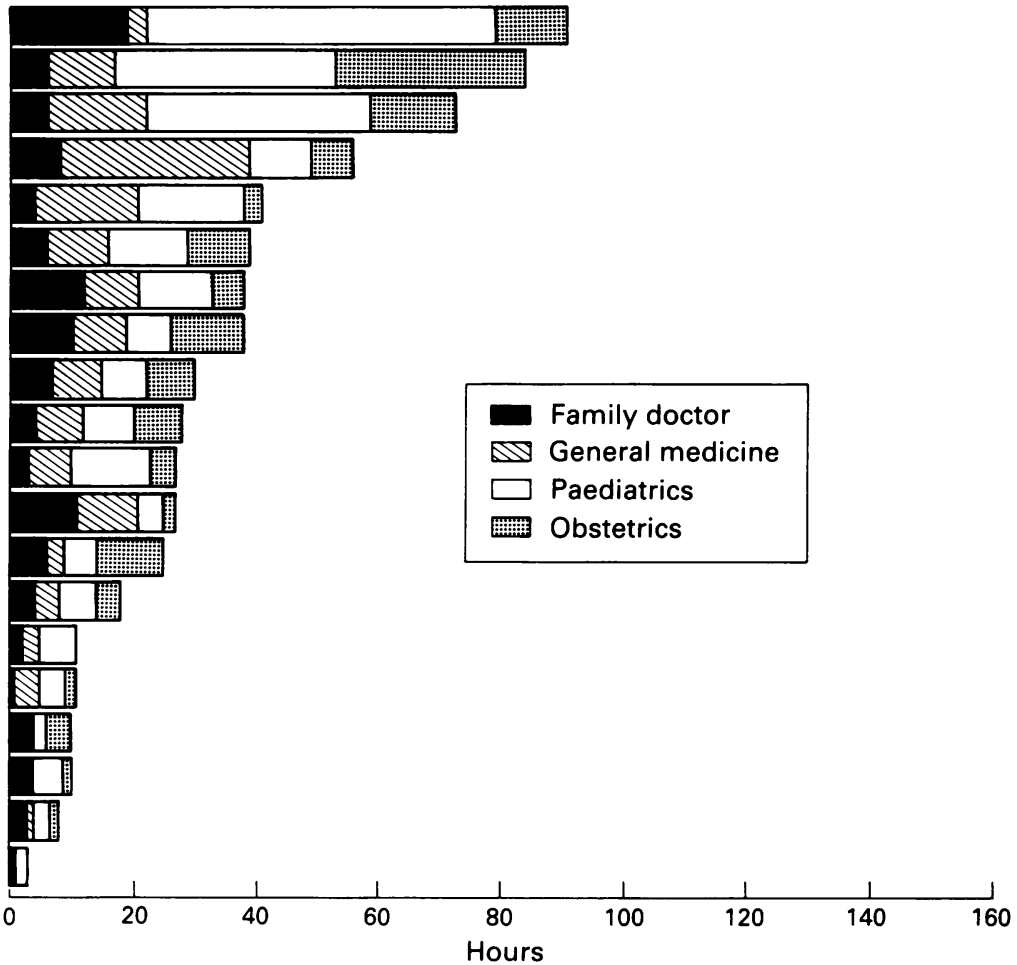

Histogram of the hours of teaching in four postgraduate courses. (Data supplied by consultant clinical geneticists.)

lectures given to nursing groups. A further survey will be required in due course.

\section{Discussion}

This remarkable variation requires explanation. The reasons are difficult to disentangle but the need for caution is emphasised by the essentially anecdotal nature of the data. Few genetic centres appear to keep systematic records of teaching carried out by all members of staff. While some under-reporting is inevitable, it is unlikely to account for differences of this order. There is no correlation apparent between the recorded hours and the number of consultant clinical geneticists, though the numbers of genetic nursing staff may be relevant in determining the number of lectures to nurses. When compared with the undergraduate data, it has to be accepted that the postgraduate data are much softer. Nevertheless, the question that needs to be asked is whether education is given a high enough priority, first by clinical geneticists against competing clinical demands with inadequate staffing, and, secondly, clinical genetics as a subject by those who organise postgraduate courses.

What form should this education take? The rank order given by non-geneticists to the core curriculum and clinical skills noted in the undergraduate report are clearly just as relevant at postgraduate level, and there are many more qualified doctors and students. While every opportunity should be taken by clinical geneticists to draw out the genetic principles from cases presented at Grand Rounds and equivalent staff meetings, their irregularity means that they cannot on their own provide an adequate basis for teaching. Similarly, occasional ad hoc lectures (usually given by visitors) are also inadequate and often too specialised, although they can provide an important contribution. There is surely a case to be made out for a short course of, say, four lectures, seminars, etc to be included as an integral part of any regular cycle of lectures in the basic courses run regularly in each discipline by the local postgraduate dean or tutor. Such lectures would cover the importance of establishing the genetic diagnosis, the obtaining of a family history, the principles of genetic counselling, and the basis of molecular genetics and cytogenetics. The final lecture would be devoted to the application to that particular specialty. Where the speed of advance in knowledge is so rapid and so evident, such an approach to postgraduate education is of prime importance in providing the necessary care for patients and their families.

I am most grateful to the postgraduate deans and clinical geneticists throughout the country for their help in providing data, and to the College Committee on Clinical Genetics of the Royal College of Physicians and its chairman Professor R Harris for their comments.

1 Royal College of Physicians. Teaching genetics to medical students: report of a working party of the Committee on Clinical Genetics. London: Royal College of Physicians, 1990. 